\title{
SOME ASPECTS OF RADIOLOGICAL INVESTIGATION OF THE HEART AND GREAT VESSELS
}

\author{
LeON Morris, M.B., F.F.R., D.M.R.D. \\ Radiodiagnostic Department, Brook General Hospital, London, S.E.1 8
}

THERE have been many advances in angiocardiography in recent years, but in this short communication I shall deal only with some of these.

\section{Technical Advances}

(i) Rapid serial films. By this means up to 6 films per second can be secured simultaneously in two planes. This allows us to study all phases of the cardiac cycle. Figs. ra, Ib and Ic demon-

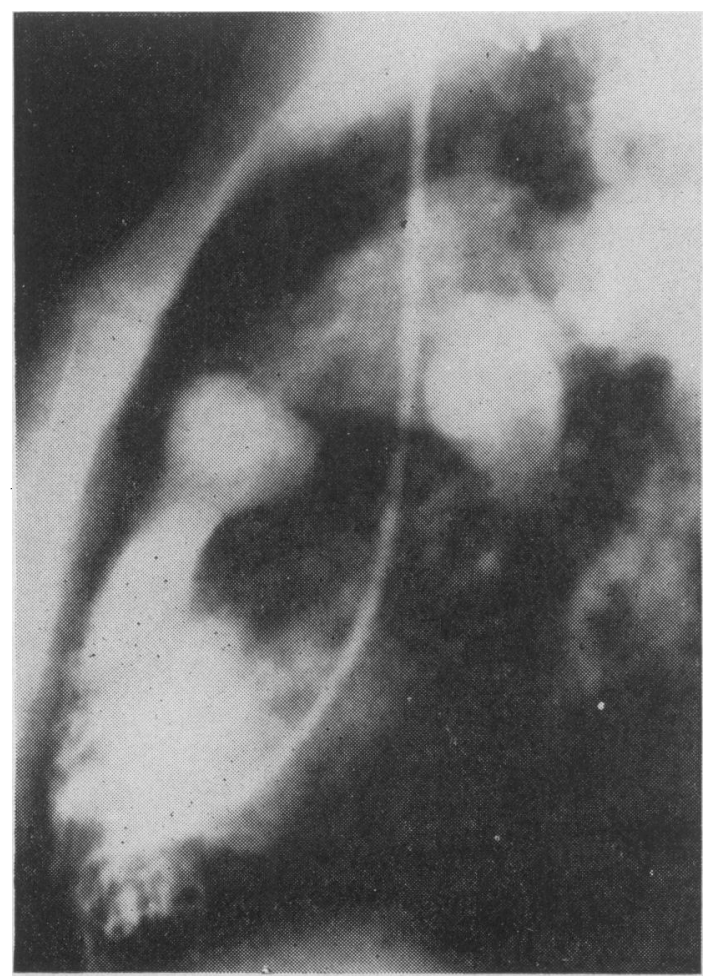

FIG. IA.-Pulmonary valvular stenosis. Catheter in the right ventricle. Systolic phase. The upward bulging of the pulmonary valves is seen.

A paper read to a Conference of Physicians of the South-East Metropolitan Region at the Brook Hospital, March 17, 1962. strate the value of this in a case of pulmonary valvular stenosis.

(ii) Intensification of the fluoroscopic image with television monitoring. This assists in screening and the placement of catheters and avoids the necessity for dark adaptation and darkening of the screening room.

(iii) Cineradiography. This gives a functional as well as an anamical assessment and used with the image intensification the radiation dose to the patient may be small.

\section{Advances in Technique}

(i) Selective injection of contrast medium. Previously contrast was injected into the basilic vein or the superior vena cava. Now the contrast is

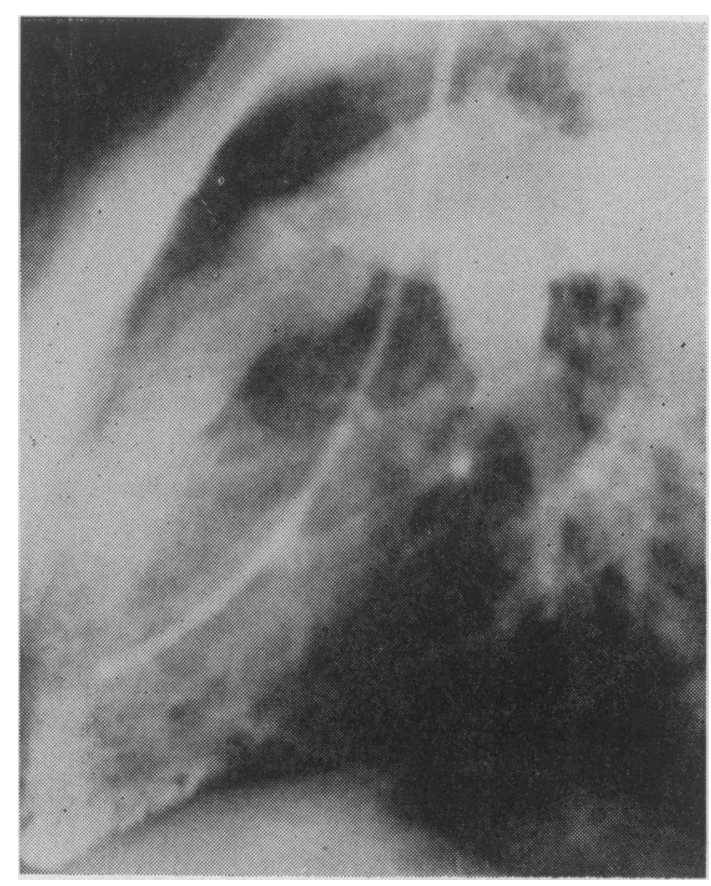

FIG. IB.-Late systolic phase. Showing the normal dilatation of the infundibulum of the right ventricle. 


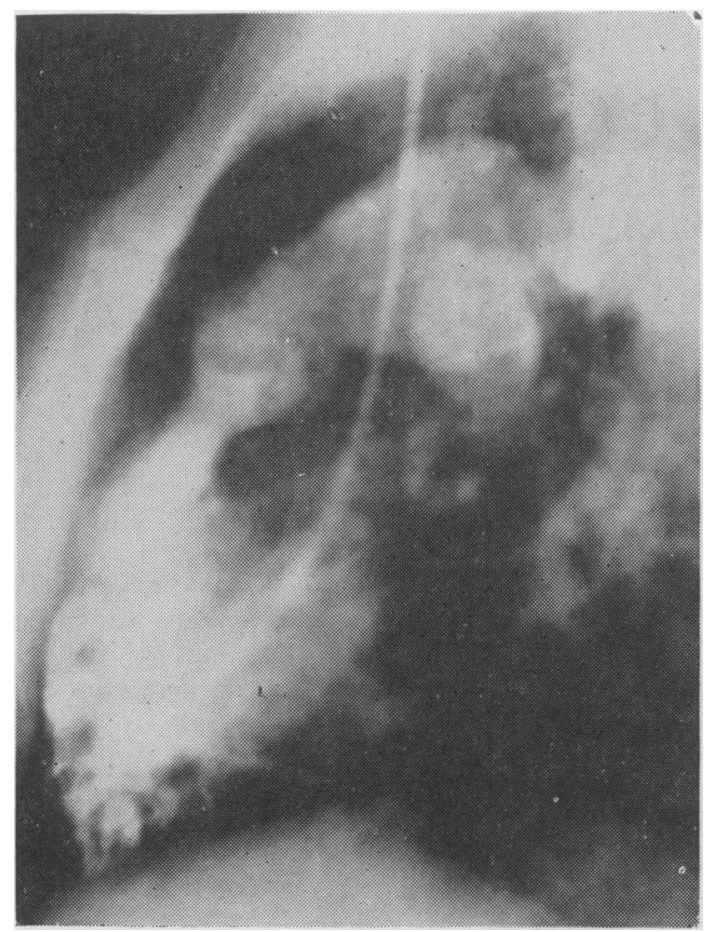

FIg. IC.-Diastolic phase. The outline of the pulmonary valves is clearly seen.

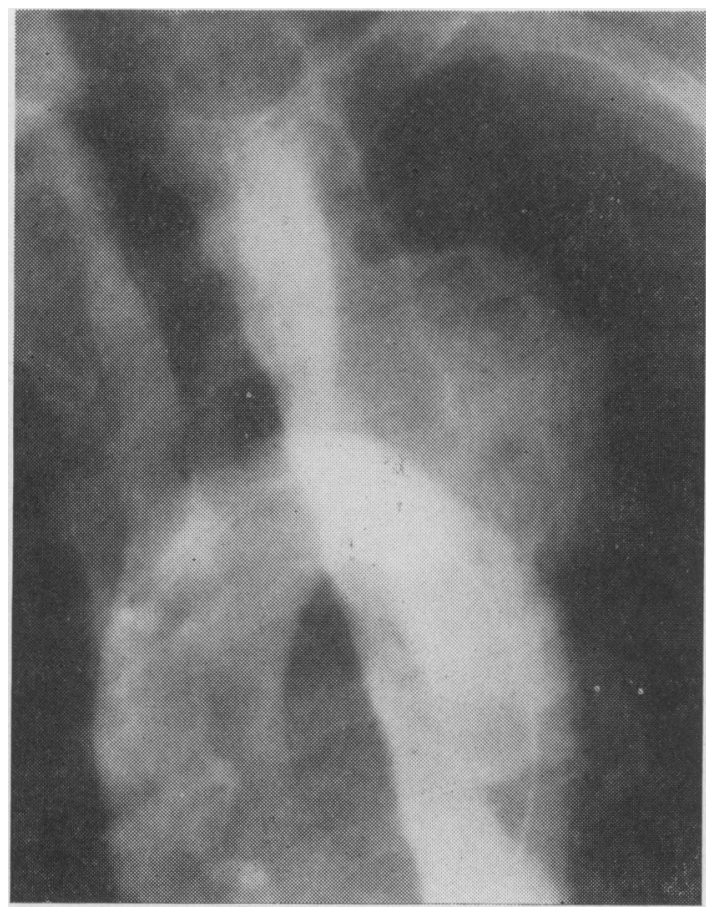

FIG. 3--Oblique film of the aortic arch demonstrating a large aneurysm of traumatic origin arising just distal to the left subclavian artery, where there is some narrowing of the aortic lumen. The catheter is seen in the aortic lumen.

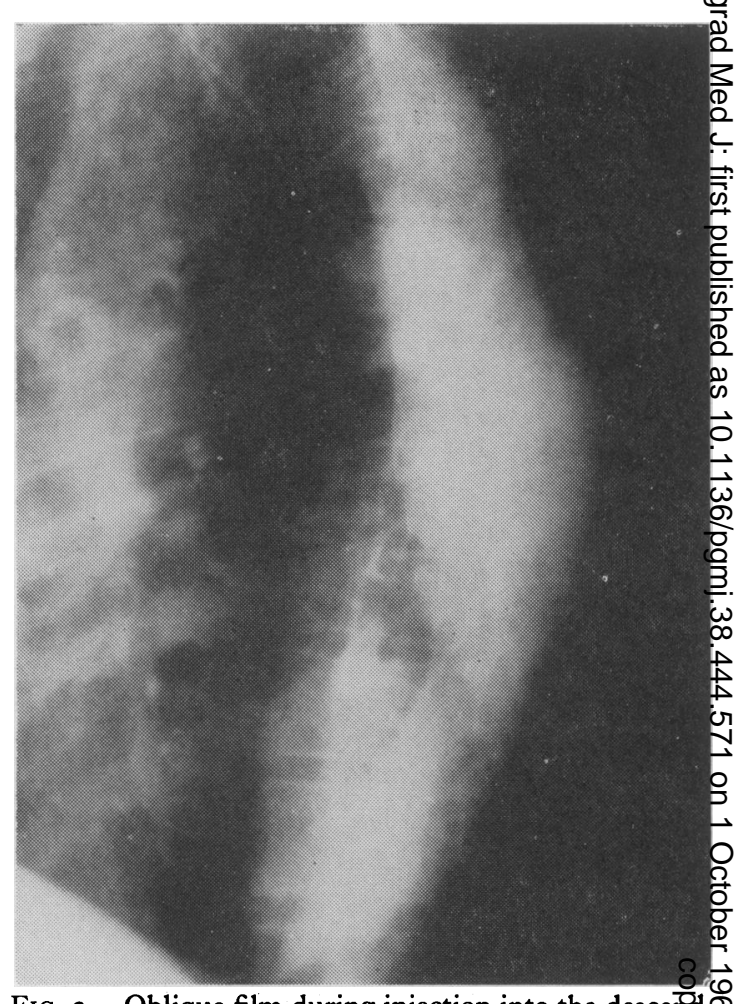

FIG. 2.-Oblique film during injection into the descerd ing thoracic aorta after femoral catheterization. fusiform aneurysm of the thoracic aorta is show

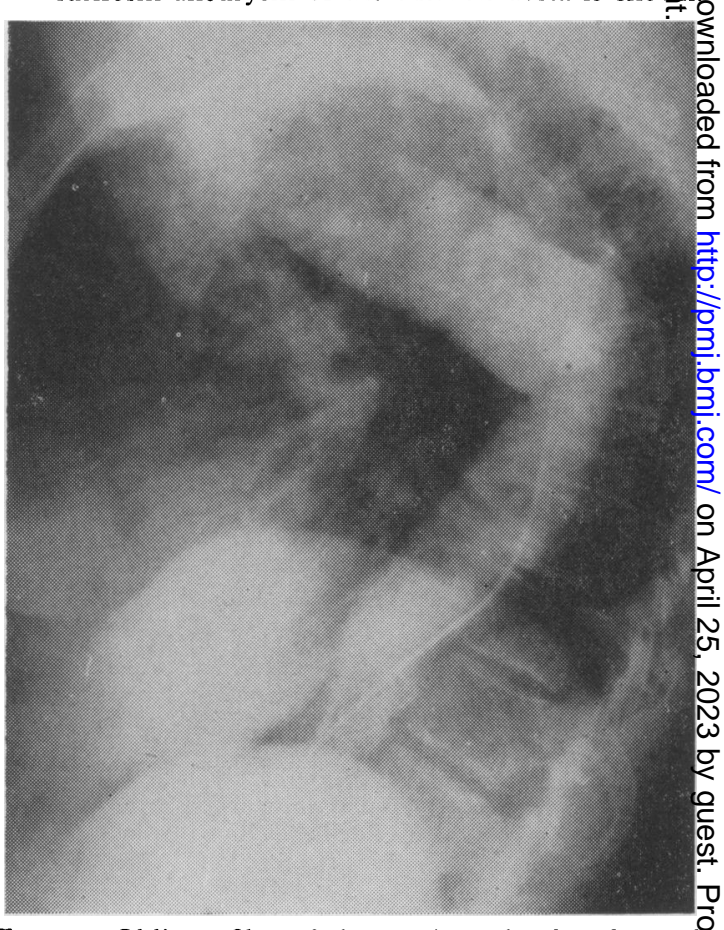

FIG. 4.-Oblique film of the aortic arch after femora욤 catheterization. The aortic lumen is distorted and? there is a wide soft-tissue space between the aortic lumen and the margin of the aortic arch. This
space indicates a dissecting aneurysm. 


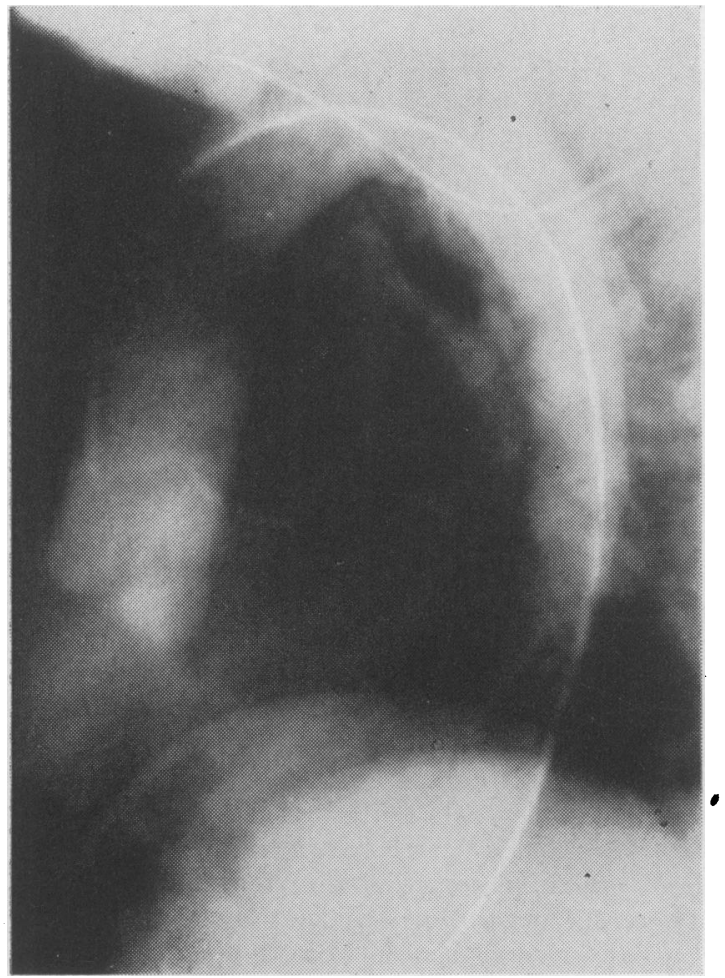

Fig. 5A.-Lateral film after injection of contrast just above the aortic valves in a case of aortic stenosis. Diastolic phase. The three aortic sinuses are seen, also the slightly thickened valve. There is some opacification of the left coronary artery. The catheter is clearly seen.

delivered as close to the site of the lesion as possible. This is demonstrated in Fig. I, where the catheter is placed in the right ventricle.

(ii) Approach to the heart and great vessels by arterial catheterization. This may be achieved by retrograde percutaneous femoral or brachial catheterization by the technique of Seldinger

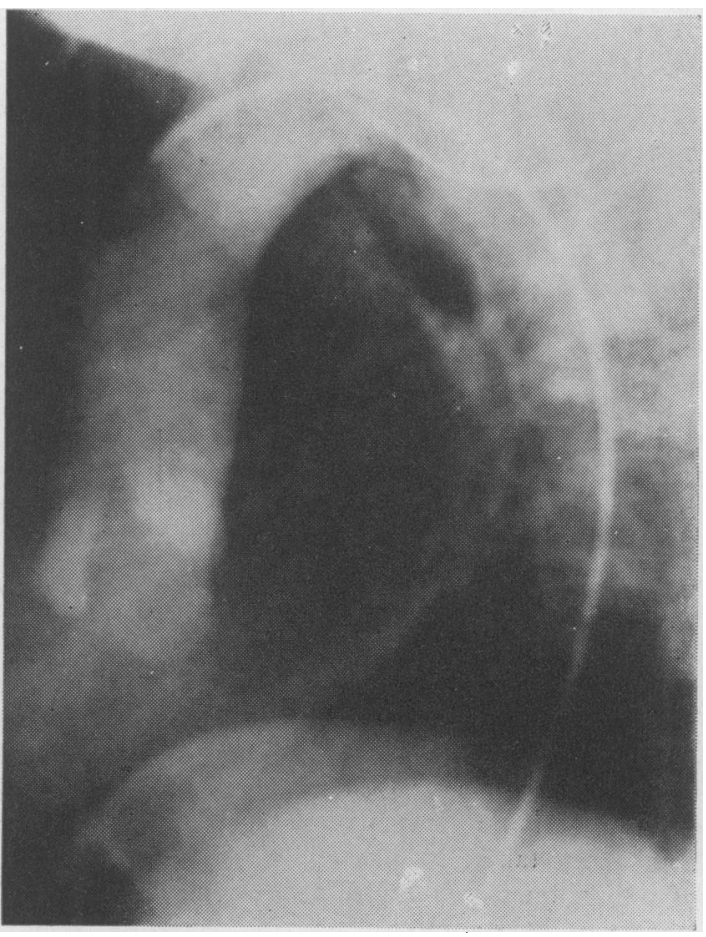

FIG. 5B.-Same case as Fig. 5a. Systolic phase. The valve cusps have not retracted normally. Nonopacified blood is seen between the cusps, the margins of which are clearly seen.

(I953), or by open arteriotomy of the brachial or femoral arteries. The catheter is passed in a proximal direction and contrast injected and films secured when the tip of the catheter is in relation to the lesion. Figs. 2 to 5 illustrate the use of this technique in lesions of the thoracic aorta and the aortic valves. The catheter can be advanced into the left ventricle to demonstrate this chamber and also the anatomy and function of the mitral valve.

\section{REFERENCE}

Seldinger, S. I. (1953): Catheter Replacement of the Needle in Percutaneous Arteriography, Acta radiol. (Stockh.), 39, 368 . 